\title{
Microsensor for Cell Force Measurement
}

\author{
Wojciech Święszkowski ${ }^{1, *}$, Waldemar T. Smolik ${ }^{1}$, Norbert Danz², Erik Forester ${ }^{2}$, \\ Jean-Pierre Kaiser ${ }^{3}$, Arie Bruinink ${ }^{3}$, and Krzysztof J. Kurzydłowski ${ }^{1}$ \\ ${ }^{1}$ Warsaw University of Technology; ${ }^{2}$ IOF Fraunhofer Gesellschaft, Jena; ${ }^{3}$ Empa St. Gallen
}

(Received: 15 January 2009. Accepted: 24 February 2010)

\begin{abstract}
Cell elasticity, motility and migration are very important for understanding various biological processes. Currently, monitoring cellular traction forces is desired as new marker to monitor cell functionality. We developed a sensor system for cell force measurement based upon a polymer microchip covered with a micropillar matrix. The spring constant is chosen to enable pillar bending by cellular forces. The microchip is read out optically. Besides a transmission image to analyze pillar bending, two different fluorescence images of dye markers can be taken sequentially. Matrix with the cells is illuminated by spatially homogenized LED radiation. The focal position during long term experiments can be maintained by means of a piezo stage in order to obtain highly resolved images from the CCD imager. Sophisticated software controls the optical system and performs image registration. First, one transmission (red) as well as two fluorescence images (blue \& green) are taken using appropriate CCD gain and integration times. Fluorescence information yields those pillar positions that are at least covered by a cell. The transmission image is used to calculate the magnitude and direction of pillars deflection. Therefore, detection of single pillar position and its deviation from a hexagonal grid is analyzed automatically, enabling a mapping of traction forces. The system containing transducer polymer chip, optics, and dedicated software analysis is a complete imaging system for image registration, single cell visualization and cellular force measurement. First results of cellular force measurements obtained are presented.
\end{abstract}

Keywords: Cell Forces, Cell Motility, Cell Imaging, Micro-Patterned Substrate, Micropost Array.

\section{INTRODUCTION}

Cell motility and migration plays a critical role in many physiological systems. Cell elasticity and the role of the cytoskeleton, a cytoplasmic system of fibers, are very important for understanding various biological processes. Cell traction force measurement can be important tool in fundamental biological research. ${ }^{1,2}$ Characterization of cells is of crucial importance in various fields of health care. Cell migration and cell contractile forces play a crucial role for the all dynamic processes such as in wound healing or bone homeostasis. Cell traction forces measurement may be useful as an additional parameter to monitor cell functionality for instance for the development of new drugs in cancer or osteoporosis therapy. The cytoskeleton maintains cell shape, enables motion and plays important roles in both intracellular transport and cellular division. Cells spread and move on surfaces to which they are

${ }^{*}$ Corresponding author; E-mail: Wojciech.Swieszkowski@inmat. pw.edu.pl adhered. A number of methods have been developed to measure cell forces. ${ }^{3}$ In most methods the deformation of the flexible substrate is taken as an index of cell traction forces but in case of continuous flexible membrane a very complex data analysis is required to quantify the forces. An alternative method of measuring traction forces is to employ a microchip with a matrix of microcantilevers. ${ }^{4-6}$ In this method, cells attach to the tops of the cylindrical cantilevers which bend in a response to cell forces. The image analysis of cantilevers deviation from the rest position enables the mapping of traction forces. The technique of using micron-sized pillars as force sensors in cell traction is now studied by several groups. ${ }^{7-9}$

This paper presents a sensor system developed for cell force measurement based upon a polymer microchip covered with a micropillar matrix (Fig. 1). A hexagonal grid of microcantilevers in the shape of cylindrical pillars was proposed as a substrate surface pattern. The spring constant is chosen to enable pillar bending by cellular forces. The deflexion of the pillars is read out optically. 


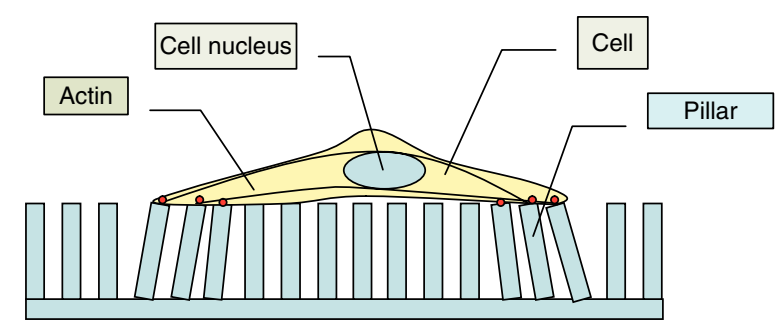

Fig. 1. Drawing of cell force microsensor with pillared substrate (side view).

\section{CELL FORCE SENSOR}

\subsection{Optical System Setup}

The biosensor is a complete imaging system for cells visualization consisting of optical system, image registration and analysis software, and microchamber for cells. The optical system (Fig. 2) designed by IOF Jena group consist of light sources-color light emitting diodes (red, green and blue), a system of lenses and prisms, the NeoFluar zoom objective with the piezo focus controller and the AVT Pike F421B monochrome CCD camera. It uses all LED illumination in order to sequentially take different images by switching the corresponding light sources only, without moving filter wheels. The system has been designed to work with a long working distance microscope objective and a commercial CCD camera. Every "fluorescence" light source comprises its own "excitation filter" that permits long wavelength radiation to reach the sample. Excitation light is reflected by a common dichroic splitter towards the sample. Red shifted fluorescence light can pass this dichroic splitter and reach the detector. In order to prevent short wavelength (excitation) radiation scattered at the sample to reach the detector, an emission filter absorbs this light. In result, by using an appropriate light source filter-splitter combination dual color fluorescence imaging can be performed using a single detector and sequential illumination.

A fluorescence imaging set up can be easily combined with classical transmission imaging. This is achieved by illuminating the sample from the bottom side in using a wavelength that transmits the splitter and the excitation filter. In result, by sequentially switching these three light sources (and adapting the camera parameters) two fluorescence and one transmission image can be taken. This yields several advantages compared to other approaches. A single detector (expensive CCD camera) is needed to ensure low costs of the system. This approach should enable a temporal resolution in the range of a few ten seconds that is much below the intended measurement every five minutes. The device works with less illumination intensity and a single plane of observation. Reduced illumination intensity reduces bleaching of fluorescent markers.

Important aspects regarding the optical design of the total system were: the homogenous illumination

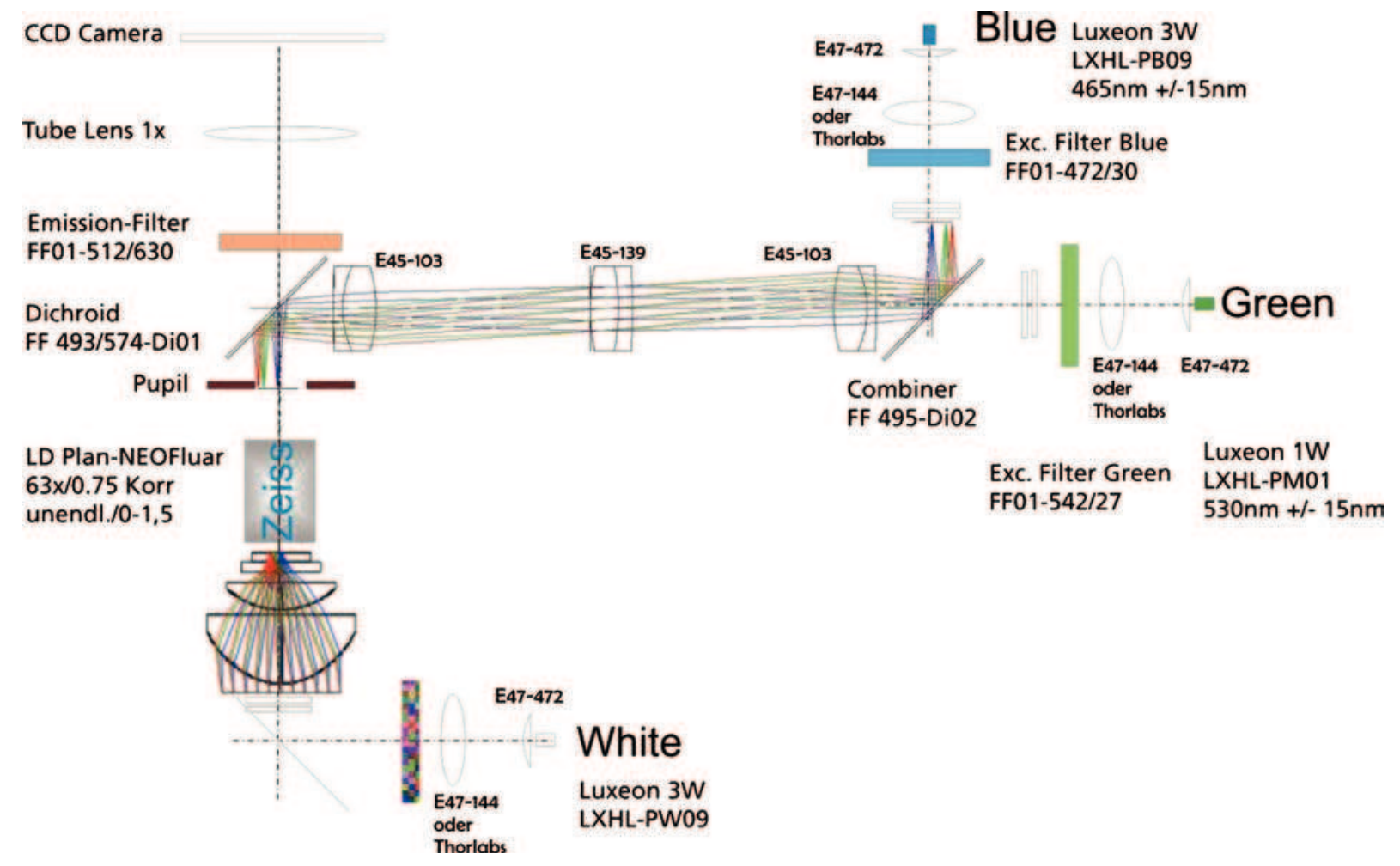

Fig. 2. Sketch of the optics design of Cell Force sensor for dual color fluorescence imaging combined with an illumination in transmission. "Blue" and "Green" are the fluorescence excitation light sources, "White" is the transmission light source. 
for fluorescence excitation, application for fluorescence illumination, homogeneous illumination for transmission imaging and fluorescence filters.

The optics to homogenize LED output is shown in the Figure 3. The light leaving the LED is collimated by means of two plan convex lenses. The collimated light passes a spectral filter to reduce the spectral bandwidth of the LED onto the desired spectral excitation range of the corresponding fluorescence marker. The two, most right, elements are crossed, double sided, cylindrical microlens arrays for homogenization. The transmission bands of all filters in the set up need to be chosen carefully to prevent any direct way of excitation light to the detector. This would reduce the image contrast and sensitivity.

The approach according to Figure 3 yields a homogeneous angular spectrum of the light source's emission. This homogeneous angular spectrum must be generated in the pupil of the objective, which will "convert" the homogeneous angular spectrum into a homogeneous spatial distribution. The application intended here requires some additional effort: First, the two light sources for fluorescence excitation must be superimposed by means of any well adapted dichroic beam splitter. Second, due to spatial reasons, relay optics is required to image the homogeneous angular spectrum into the pupil of the microscope objective. For transmission illumination the sample is directly illuminated without prior passing the objective. Therefore, some sort of Fourier optics needs to be introduced in order to convert the homogeneous angular spectrum into a homogenous spatial illumination inside the sample. Figure 2 depicts this optical set up. A set of two plan convex lenses has to be adjusted near the lower cover glass of the sample holder. Note that this illumination system has to be brought in close proximity to the sample, thus requiring a carefully designed mechanical housing as well as a specified thickness of the polymer samples. Commercially available fluorescence filter sets from Semrock have been used. The spectral properties are depicted in Figure 4. Note the two separate observation bands (red

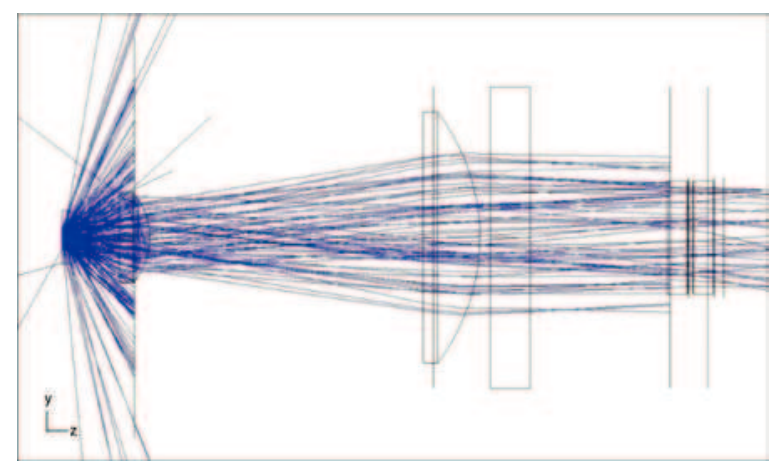

Fig. 3. Detailed ray tracing image of the LED collimation and homogenization. The optical elements shown from left to right are: LED equipped with half ball lens, two planconvex lenses to collimate the LED radiation, fluorescence excitation filter, and two crossed double-sided cylindrical lens arrays.

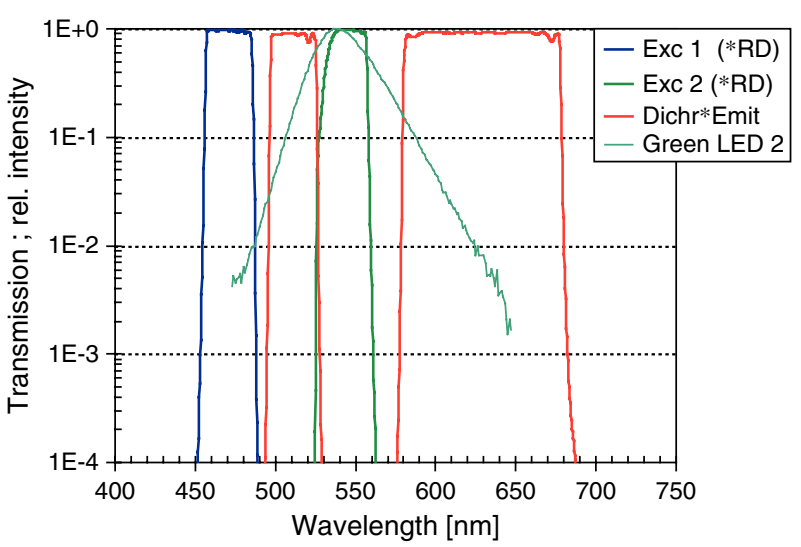

Fig. 4. The transmission of the two excitation filters (thick blue and green lines) as well as the product of emitter transmission and dichroic splitter reflectivity (thick red line). The green LED emission spectrum is given as thin green line for comparison.

line) that correspond to the two different spectral excitation bands.

The total optical system is shown in Figure 5. In order to operate the system, additional electronic equipment is necessary. This includes the piezo control unit comprising a RS-232 interface for focus fine adjustment, as well as a power supply for the LED light sources. The latter has been set up at IOF and contains a TTL inputs to switch the different sources on or off. The brightness of the different LED can be set manually only. This adjustment should be done at the beginning (initial adjustment) of an experiment and shall not be changed later on. Therefore, manual brightness control seemed to be sufficient.

\subsection{Pillared Microchip}

The specialized microchamber (Fig. 6) has been designed to enable nutrition supply and temperature control of the

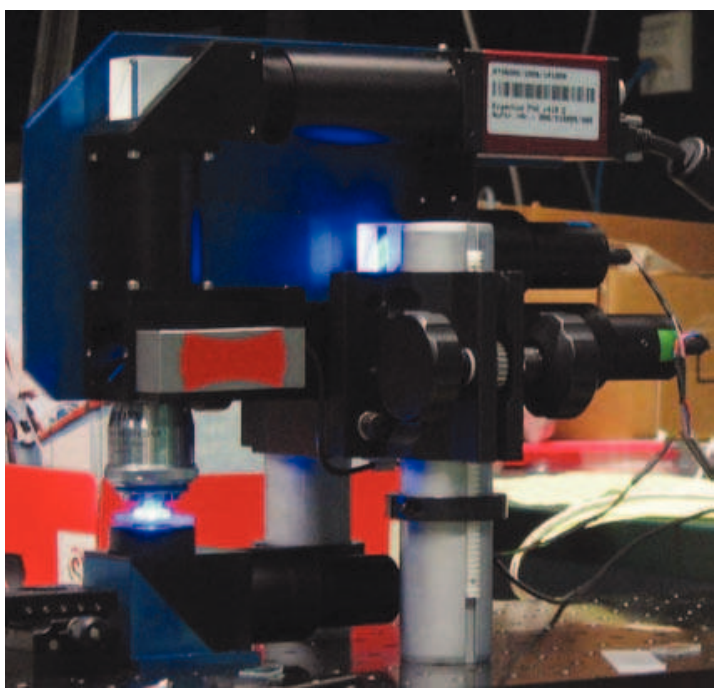

Fig. 5. The optics system without housing plate. 


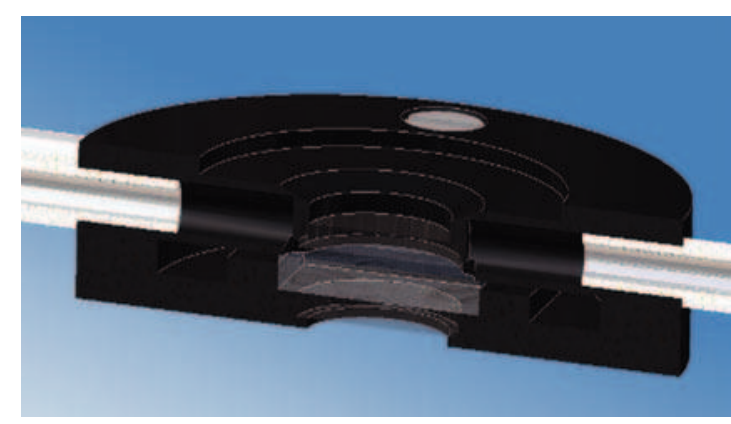

Fig. 6. Flow cell adapted to the near condenser optics for bottom side illumination. The cell contains fluid connections to supply culture medium as well as connections for water based temperature control.

living cells. The chamber contains fluid connections that enables culturing of living cells an aqueous solution in time of measurements. The so called "flow cell" supports the flexible pillar matrix-injection moulded polymer ${ }^{10}$ made at IFAM Bremen. The glass windows from the top and bottom enables microstructure observation.

Several contradictory requirements on chemical, mechanical and optical properties should be met by the micropillar matrix. The material should be transparent to enable the registration of transmission images of pillar array and shouldn't manifest fluorescence in case of cell fluorescence imaging. The applied chemical surface coatings should enable cell adhesion to pillar tops and prevents adhesion to pillar sides. From two tested materials Sylgard ${ }^{\circledR} 184$ (polydimethylsiloxane) and Elastollan ${ }^{\circledR}$ 1180A50 (polyether-based thermoplastic polyurethane), the latter was selected for pillar matrix. The surface modifications either with polyethylene glycol (PEG) or hydroxylethyl methacrylate (HEMA), hexamethylene diamine (HMD) or chitosan (CT) by using UV irradiation, gamma irradiation (GI) and interfacial modification (IM) were tested. ${ }^{11}$ The surface of top of pillars was modified by IM with PEG to increase cell adhesion. The sides of pillars were oxidized with hydrogen peroxide $\left(\mathrm{H}_{2} \mathrm{O}_{2}\right)$ and then impregnated with PEG 400 to decrease the cell adhesion ${ }^{12}$ and to prevent the migration of the cells between pillars or even to the bottom of the pillar mat.

The mechanical model of a pillar was elaborated (Fig. 7) using finite element method (FEM). The simulations of cell-pillar interaction were performed. The characteristics of a pillar deflection versus a material stiffness, pillar height and shape were generated. The obtained mechanical characteristics allow the selection of a material for a substrate and the proper selection of pillar geometry for a given material. Different geometrical dimensions were considered to find optimal bending characteristic i.e., the bending of few micrometers. The aspect ratio height/diameter is important for the moulding. By increasing this value the difficulties to remove the pillars out of the form also increases.

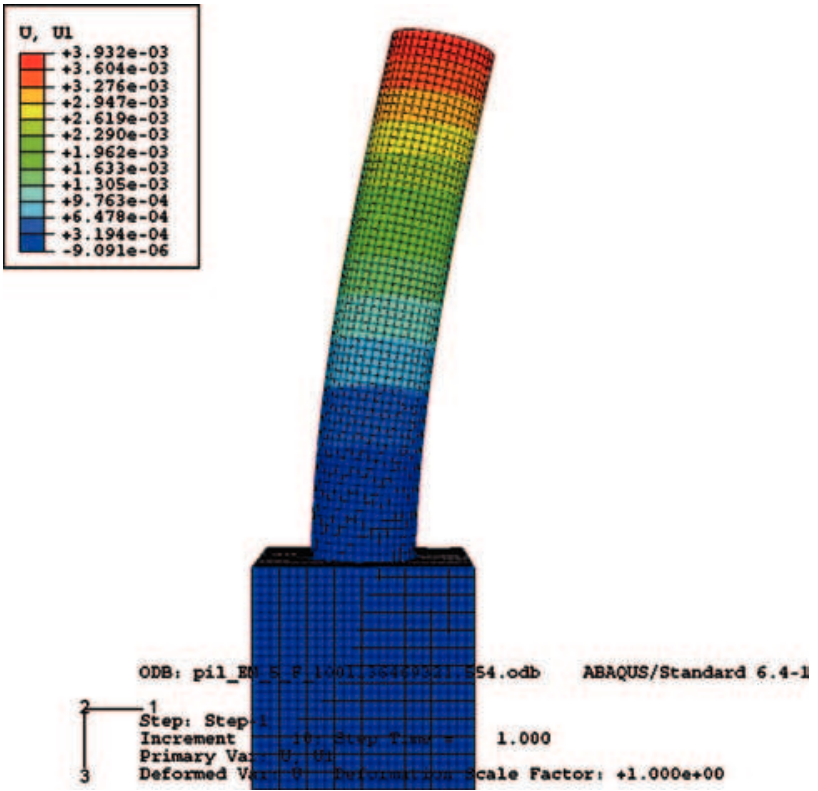

Fig. 7. Micropillar bending $[\mu \mathrm{m}]$ calculated using FEM analysis. Bending force $F=10 \mathrm{nN}$. Pillar diameter $d=5 \mu \mathrm{m}$, height $h=25 \mu \mathrm{m}$. Young modulus $E=5 \mathrm{MPa}$.

\subsection{Image Registration Software}

The "CellForce Grabber" has object oriented structure (Fig. 8) and is created in .Net programming environment. The software controls the optical system consisting of light sources (colour LEDs), a NeoFluar objective with a piezo actuator and a CCD camera. The LEDs supplier was built with 3 TTL inputs for external control. The National Instruments NI-6501 USB I/O device is used as an interface to the LEDs supplier. The CNIDAQmx class controls the light sources through NI-DAQmx 8.5 library. The focus control is encapsulated in CNVSerialPort class. The Zeiss PlanNeofluar objective is applied in the optical system. The objective coarse positioning is performed by user after inserting a substrate with a cell culture using mechanical transmission. The ZEISS piezo focus attachment, which adds ultrafine objective focusing, is used for an extremely fine Z-focus capacity. The NV40/1voltage amplifier from Piezo Jena supplies the voltage on the piezo element. The communication with the NV40/1 voltage amplifier is performed by serial RS-232 line. The software sends text commands to set given voltage value $(-10$ to $+150 \mathrm{~V})$. The monitor output receives a signal from the measurement system and with it, the information of the current position. The focus fine regulation is performed using software control panel and two sliders for fine and ultrafine objective position adjustment. The NV40/1 piezo controller work in so called "open loop" mode i.e., without position measurement and hysteresis compensation. The focus should be set always by starting from 0 Volts position. NV40/1 amplifier is slow device because it responses at least after $400 \mathrm{~ms}$. The voltage change should be done 


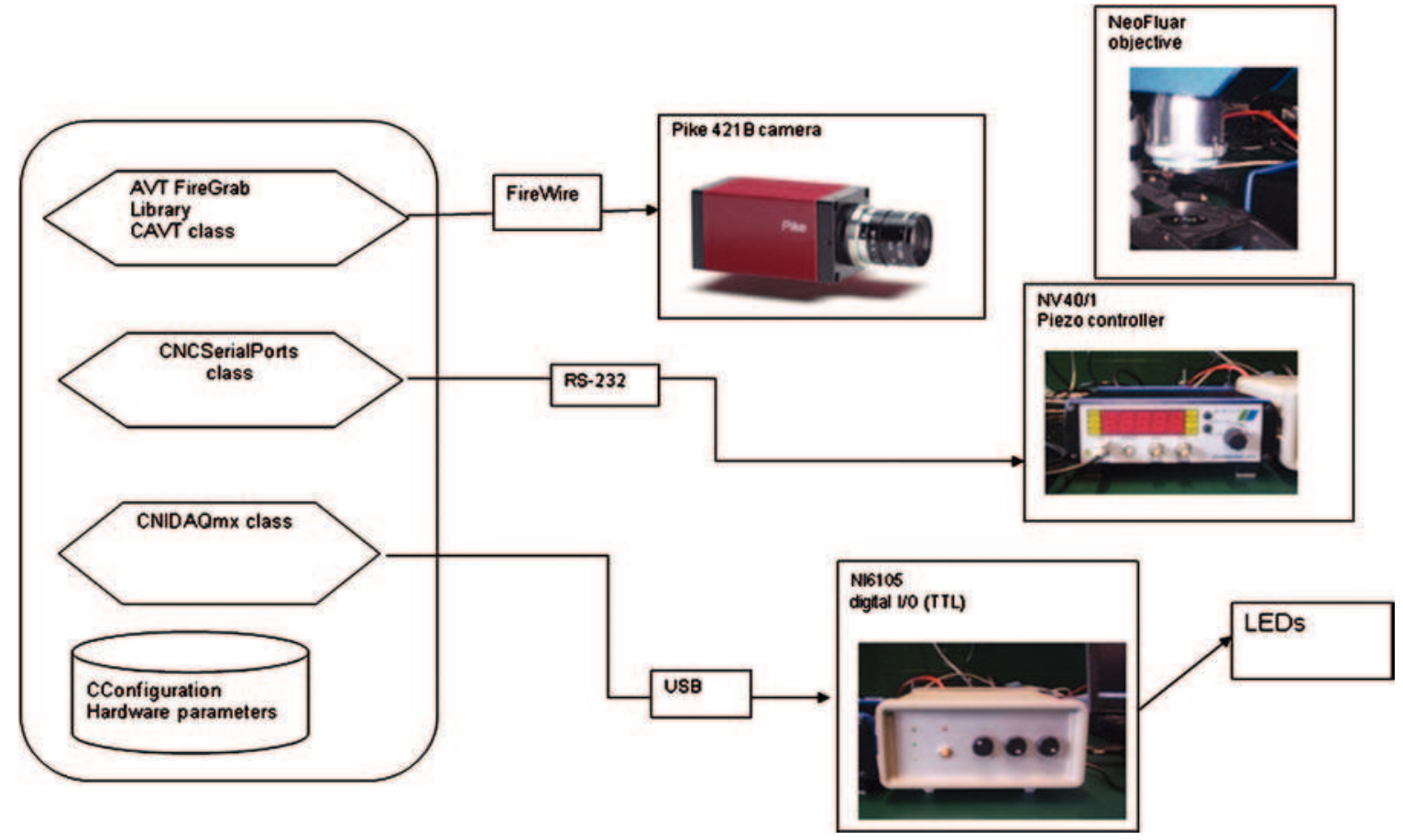

Fig. 8. The object oriented structure of the optical system control.

using small steps to avoid a damage of piezo. Because of the hysteresis the automatic focus procedure was elaborated. The procedure scans linearly the whole range of positions using limited number of steps and calculates the contrast in the rectangular region in the centre of an image. The sub range with maximal contrast value is selected and search is repeated with smaller steps. The procedure is repeated iteratively until there is no contrast increase. The position with maximal contrast value is selected.

The images are recorded by AVT Pike F421B monochrome camera with high resolution $(2048 \times 2048$ pixel matrix) and high-quality CCD sensor (CCD cell size $7.4 \mu \mathrm{m} \times 7.4 \mu \mathrm{m}$, resolution depth from 8 bits to 16 bits). The FireGrab library is used for interfacing with the Pike camera hardware by IEEE 1394b standard. The camera control and image registration are encapsulated in the CAVT class. The group of methods allows to set camera parameters like image resolution, pixel binning, gain, shutter, shutter speed and brightness. The preview mode enables the setup of these image parameters. For green and blue light used for registration of cell fluorescence images image matrix of size $1024 \times 1024 b$ should be selected. In this mode camera performs $2 \times 2$ pixels binning i.e., combines neighbouring pixels while being read out. This gives a brighter image and improves the signal to noise ratio. The CCD camera shutter time should be increased for cell fluorescence images. The focus should be adjusted for fluorescence images. The performed tests showed that the focus position should be different for transmission and fluorescence registration to obtain sharp images. In registration mode the software will automatically switch the position of objective according to the values stored in the configuration file. The second group of methods enables to register images in "one shot" or continuous mode synchronized with configurable timer. The continuous mode allows to observe cell movements. The user has the possibility to select image acquisition time and frame rate. The frame rate is specified by time interval between images. Typical interval between the following images is 5 minutes. The acquisition time may be set from one minute to several hours. The image may be stored in bitmap or jpeg format.

The other group of classes provides the user interface in form of the graphical panels with the controls (Fig. 9). The Program class encapsulates the application process and execution processes. The MainWindow class proceeds the user requests and the image display. The last group of class provides the program configuration. The configurable parameters are: ports for communication with the hardware, camera parameters, acquisition parameters like a frame rate, time, and file format and data storage location.

The "CellForce Grabber" registers the transmission image and fluorescent images with different camera settings. Regardless of the image type, the acquisition procedure is the following:

- set camera parameters (image matrix size, gain, shutter time, brightness),

- adjust focus (objective position),

- switch on LED,

- register image,

- switch off LED. 


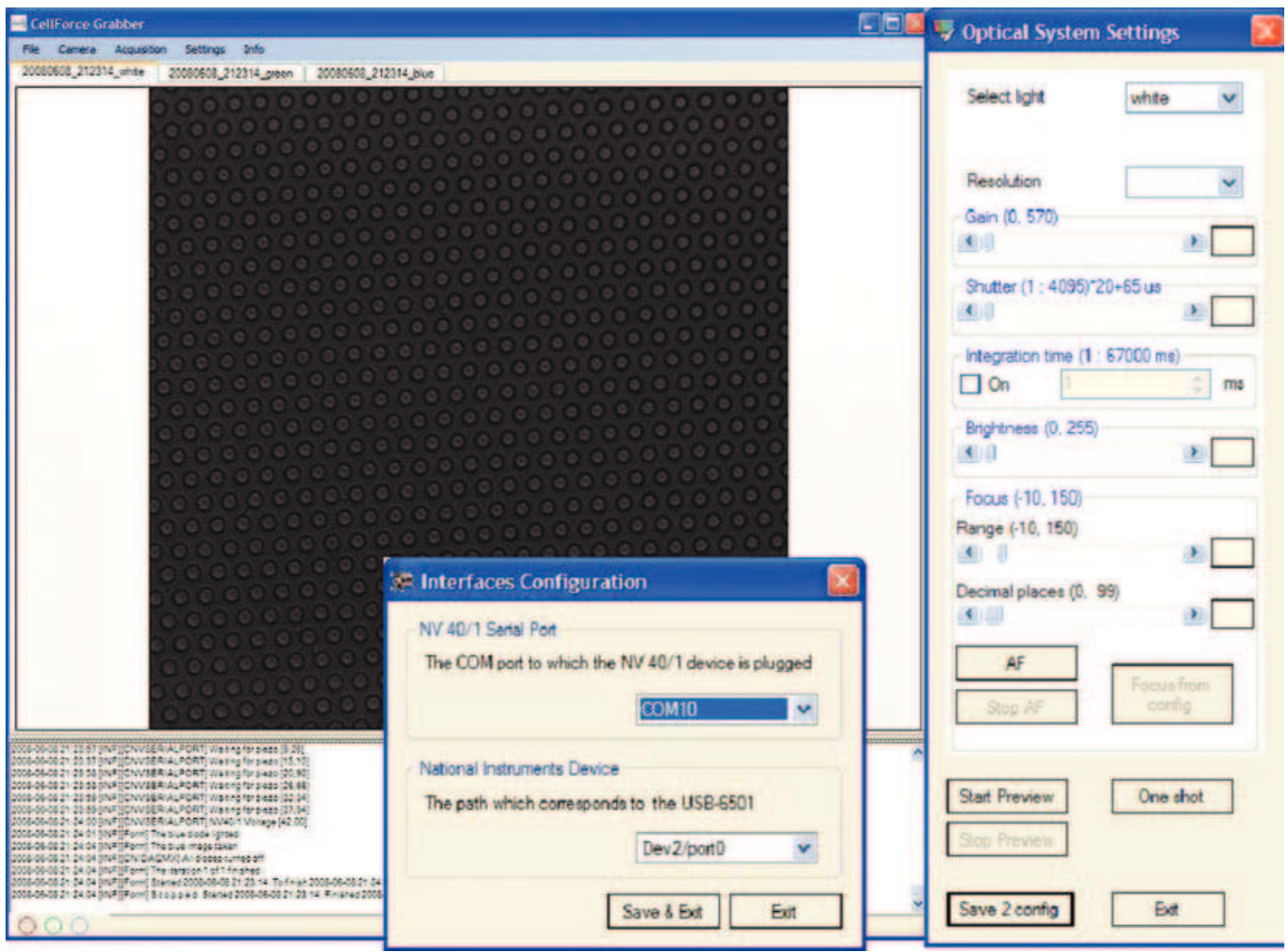

Fig. 9. CellForce Grabber graphical interface. Main and configuration windows. Transmission image of the substrate with hexagonal structure of pillars.

For a transmission image of pillared structure, the image matrix is $2048 \times 2048$ pixels; the white LED is switched on. For a cell fluorescence image, the image matrix is $1024 \times 1024$ pixels with pixel binning; the green or blue LED is used accordingly. The three images are registered in the order: white, green and blue. The optical system together with the software was tested and worked correctly.

\subsection{Image Processing}

An appropriate image analysis should be applied to calculate cell forces on the base of pillared microstructure images obtained from the microsensor. A dedicated image processing algorithm was elaborated for a hexagonal layout of pillars described in detail in Ref. [13].

The cells transmit the contraction forces to the microchip surface resulting in a bending of the cylindrical pillars aligned in a hexagonal grid. The tops of pillars appear on a transmission image of substrate as spots (Fig. 10). The spot shift from a grid nodes indicates a pillar bending and is proportional to the cell contraction force. The calculation of pillar bending requires the information of the base position of pillar. The different methods may be used to determine the reference position of pillars e.g., a registration of an image of the bottom of pillars. ${ }^{6}$ The method of calculating a reference grid using pillars unoccupied by cells (or not bended) was

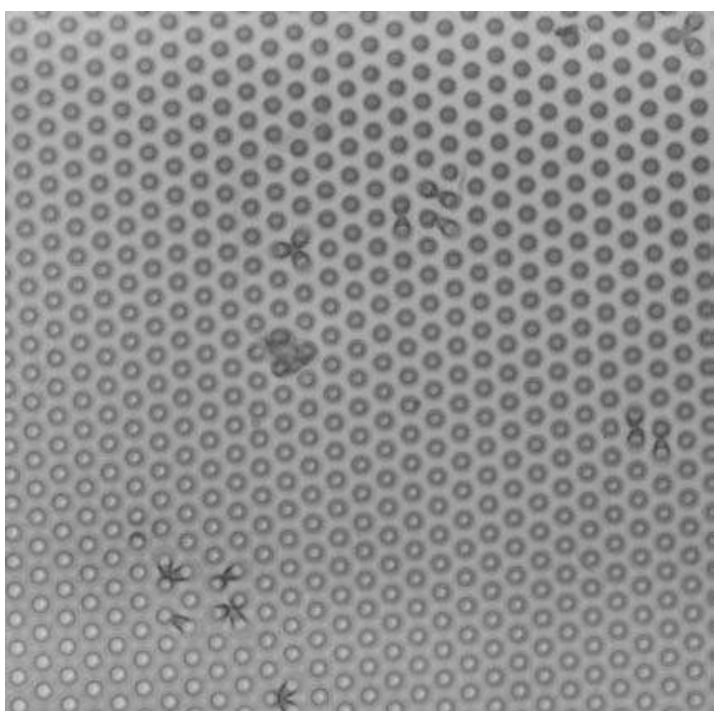

Fig. 10. Transmission image of the substrate obtained from CellForce sensor. The cylindrical pillars appear as spots. The pillar diameter is $5 \mu \mathrm{m}$, pillar height is $25 \mu \mathrm{m}$. The center-to-center distance is $10 \mu \mathrm{m}$.

selected for implementation. This method eliminates the need of the registration of any additional reference images.

The image processing consists of the following steps. The spots (tops of pillars) are identified on a transmission (red) component (Fig. 12(a)) of a cell image (Fig. 11) obtained from the microsensor. The images from the 


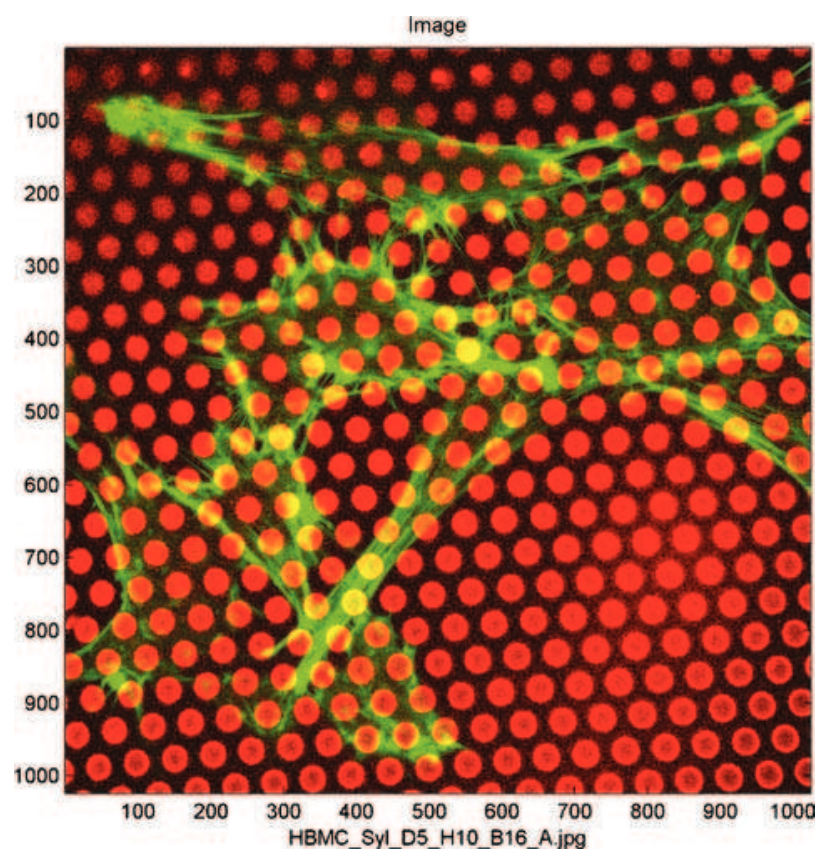

Fig. 11. Image of pillared substrate with human bone marrow cells (HBMC) obtained from Cell Force sensor. The image is a sum of transparent image of the pillar matrix saved as red component and fluorescence image of cells saved as green component of RGB bitmap. Pillar diameter $5 \mu \mathrm{m}$, pillar height $10 \mu \mathrm{m}$. Center-to-center distance $10 \mu \mathrm{m}$.

microsensor are noisy with non uniform illumination and a non uniform background due to a deformed substrate made of a flexible material. The images are denoised by the application of an average and median filters. A background is eliminated, small artefacts are removed, and a shape of pillar spots is corrected by morphological operations. Because the size and the shape of a searched spots are known a template matching algorithm was selected for spots identification. The Hough transform for circles was applied. ${ }^{14}$

The pillars are classified into two groups bent or unbent. The neighbourhood of each individual spot consisting of the six neighbour spots is analyzed. The structures are classified according to the number of detected neighbour spots and to the fit to the hexagonal layout. The unshifted spots form lines of a hexagonal lattice.

The reference hexagonal grid is identified using unshifted spots. The method eliminates the need of recognition of regions of pillars covered by cells. The hexagonal grid identification is performed by a least square optimization. The least square norm is minimized by linear search performed in four-dimensional space where the coordinates are: rotation angle, grid size, $\mathrm{X}$ and $\mathrm{Y}$ position. The search is limited to the small range in all dimensions because the approximate values of all parameters are known. The approximate rotation is found in previous step of algorithm. The size of grid is known. The range of $\mathrm{X}$ and $\mathrm{Y}$ position is limited by the size of grid. Because the substrate material is not stiff and can be stretched or squeezed the assumption that the array of microposts is a
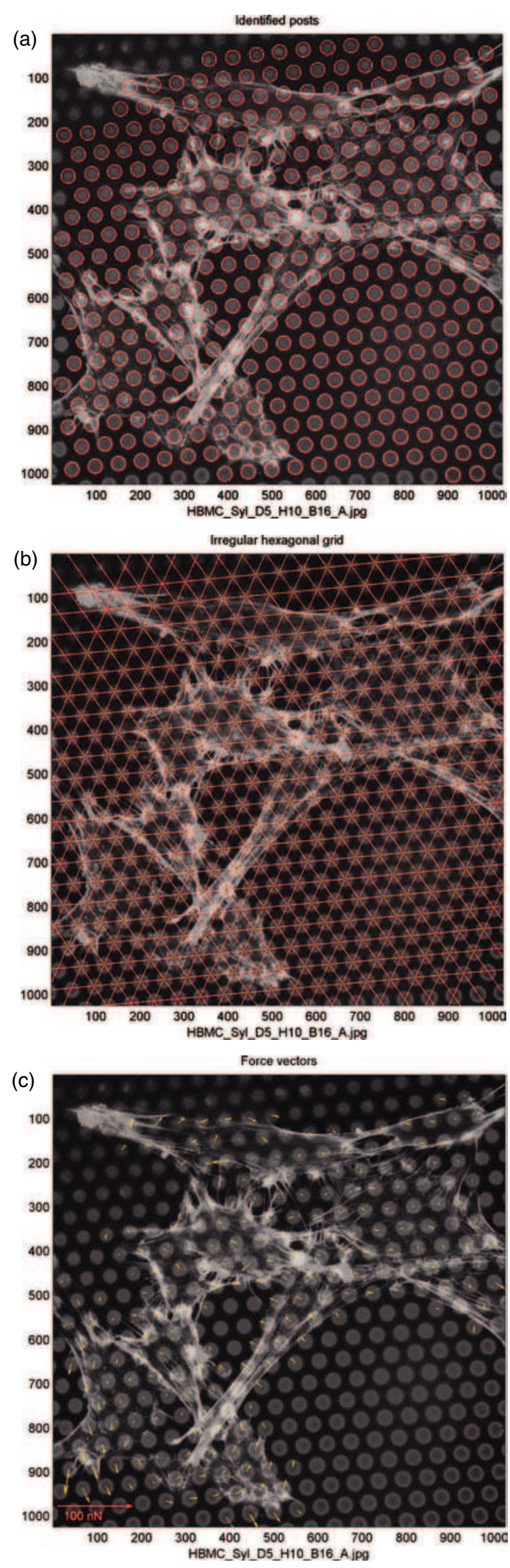

Fig. 12. Cell force identification for Sylgard pillar matrix with HBMC, (a) Identified pillar spots, (b) Fitted irregular hexagonal grid, (c) Identified bending forces. 
regular hexagonal grid is not correct. The fit to irregular grid is performed. The spots are assigned to the lines of hexagonal grid using the information from previous step. Every line of the hexagonal grid in three directions is fitted to the appropriate row or column of pillar spots using a linear regression procedure. The least square solution for the cross point of three lines with angles of 60 and 120 degrees between them is taken as grid node i.e., the rest position of pillar. The algorithm itself finds bent pillars and eliminates them from calculating the position of hexagonal grid. The tests on real images show that the enhanced algorithm performs well for irregular grid (Fig. 12(b)).

The spots shifts relative to the reference hexagonal grid are calculated. The force vector direction and value is calculated using a mechanical model of pillar bending (Fig. 12(c)). The pillars work as simple cantilevers so that their deflection, in a linear range of deformation, is proportional to the focal force applied by the cell ${ }^{15}$ according to formula

$$
F=3 / 4 \pi E \frac{r^{4}}{L^{3}} \Delta x
$$

where $r, L$, and are the radius, length and deflection of the pillar respectively, and $E$ is the Young's modulus.

\section{CONCLUSIONS}

The low cost sensor for cell force imaging was built. The optical system does not require laser scanning. The microchamber for living cells and pillared matrix enables culturing of living cells. The dedicated software provides optical system control, image registration and cell visualization. The transmission images of pillared microchip as well as fluorescence cell images are registered. The elaborated image analysis enables cellular force measurement. The first tests of device were performed and sets of images of fixed cells were analysed. The first time-lapse video experiments. using living cells however revealed that no cell forces with the current set-up could be detected. This can be explained by limits of the detection method, i.e., the very small pillars deflections are below the accuracy of the method due to the fact that forces of living cells are below the expected range, i.e., that pillar material was too stiff for the tranduced forces. There may be several other reasons of a lack of detection of cell influence with a pillared substrate. Poor cell adhesion and poor cell spreading was also observed. The experiments will be repeated for new pillar materials and modified coatings at the top and the sides of pillars.

\section{References and Notes}

1. S. Huang and D. E. Ingber, Nat. Cell Biol. 1, E131 (1999).

2. J. Y. Wong, J. B. Leach, and X. Q. Brown, Surf. Sci. 570, 119 (2004).

3. A. K. Harris, Jr., J. Biomech. Eng. 106, 19 (1984).

4. C. G. Galbraith and M. P. Sheetz, Cell Biology Proc. Natl. Acad. Sci. USA 94, 9114 (1997).

5. J. L. Tan, J. Tien, D. M. Pirone, D. S. Gray, K. Bhadriraju, and C. S. Chen, PNAS 100, 1484 (2003).

6. C. A. Lemon, N. J. Sniadecki, S. A. Ruiz, J. L. Tan, L. H. Romer, and C. S. Chen, Mech. Chem. Biosyst. 2, 1 (2005).

7. C. M. Cesa, N. Kirchgeßner, D. Mayer, U. S. Schwarz, B. Hoffmann, and R. Merkel, Rev. Sci. Instrum. 78, 78:034301 (2007).

8. N. Tymchenko, J. Wallentin, S. Petronis, L. M. Bjursten, B. Kasemo, and J. Gold, A Novel Biophysical Journal 93, 335 (2007).

9. R. Zaidel-Bar, M. Cohen, L. Addadi, and B. Geiger, Biochem. Soc. Trans. 32, 416 (2004).

10. N. Salk, J. Haack, J. P. Kaiser, A. Bruinink, and A. Rota, European Society for Precision Engineering and Nanotechnology-EUSPEN-: European Society for Precision Engineering and Nanotechnology, EUSPEN 2008, 10th Anniversary International Conference, Kongresshaus Zürich, May, Euspen, Bedford (2008), Vol. 2, pp. 587-591.

11. P. Alves, J. F. J. Coelho, J. Haack, A. Rota, A. Bruinink, and M. H. Gil, Eur. Polym. J. 45, 1412 (2009).

12. P. Alves, J. P. Kaiser, J. Haack, N. Salk, A. Bruinink, H. C. de Sousa, and M. H. Gill, Colloid. Polym. Sci. 287, 1469 (2009).

13. W. T. Smolik, W. Święszkowski, K. J. Kurzydłowski, A. Bruinink, and N. Danz, Under Review in biocybernetics and biomedical engineering, Fundamentals of Digital Image Processing, edited by A. Jain, Prentice-Hall (1989).

14. A. Ganz, M. Lambert, A. Saez, P. Silberzan, A. Buguin, R. M. Mege, and B. Ladoux, Biol. Cell 98, 721 (2006). 